\title{
Intraductal papillary mucinous neoplasm of the pancreas
}

A 53-year-old lady presented with a 2-month history of recurrent attacks of moderate intensity epigastric pain radiating to the back, which was relieved with oral analgesics. There was no fever, anorexia or weight loss. There was no history of smoking or alcohol consumption. Clinical examination was unremarkable. Laboratory investigations revealed - hemoglobin $10.4 \mathrm{~g} / \mathrm{dL}$, serum amylase $128 \mathrm{U} / \mathrm{L}$ (normal $<200 \mathrm{U} / \mathrm{L}$ ), serum alkaline phosphatase $468 \mathrm{U} / \mathrm{L}$ (40-125) and CA19-9 307 U/L (<33). Abdominal computed tomography (CT) scan (Fig. 1) revealed a $5 \mathrm{~cm} \times 4 \mathrm{~cm}$ mass involving the head and uncinate process of pancreas, dilated biliary and pancreatic ducts with no evidence of distant metastasis. A side-viewing duodenoscopy (Fig. 2) revealed gaping of both the major and minor papillae, plugged with mucin, producing the classic "fish-eye appearance" suggestive of intraductal papillary mucinous neoplasm (IPMN) of the pancreas. Fluid from the pancreatic duct showed atypical cells. The patient underwent Whipple's resection. Histopathology examination revealed an IPMN of pancreas (main duct type) with moderate dysplasia. The resection margin and regional lymph nodes were free of tumor.

Recurrent abdominal pain associated with a pancreatic mass is a common problem. The common differential diagnosis would include an adenocarcinoma or an inflammatory mass associated with chronic pancreatitis. ${ }^{1}$ In our patient, side-viewing duodenoscopy was done to look for duodenal infiltration by a mass. The classic fish-eye appearance suggested the diagnosis of IPMN which was confirmed by microscopic examination of pancreatic juice. Unlike adenocarcinoma of pancreas, complete resection of an IPMN augurs a much better prognosis. Endoscopic ultrasound is being increasingly used to diagnose IPMN. EUS findings that may predict malignant potential of an IPMN of the pancreas are: location of the lesion in the main duct, size of lesion $>3 \mathrm{~cm}$ (if located in the branch duct) and presence of a mural nodule $>5 \mathrm{~mm}$ size. ${ }^{2}$ Follow up is required because of the association with extrapancreatic malignancies, notably gastric and colonic cancers.

Ebby Simon $^{1} \cdot$ A. J. Joseph ${ }^{1} \cdot$ Lisa Choudhrie $^{2} \cdot$ Anu Eapen $^{3} \cdot$ Frederick Vyas ${ }^{4} \cdot$ V. Sitaram ${ }^{4} \cdot$ B. S. Ramakrishna $^{1} \cdot$ Ashok Chacko $^{1}$

Departments of ${ }^{1}$ GI Sciences, ${ }^{2}$ Pathology, ${ }^{3}$ Radiodiagnosis, and ${ }^{4}$ Surgery, Christian Medical College, Vellore 632 004, India

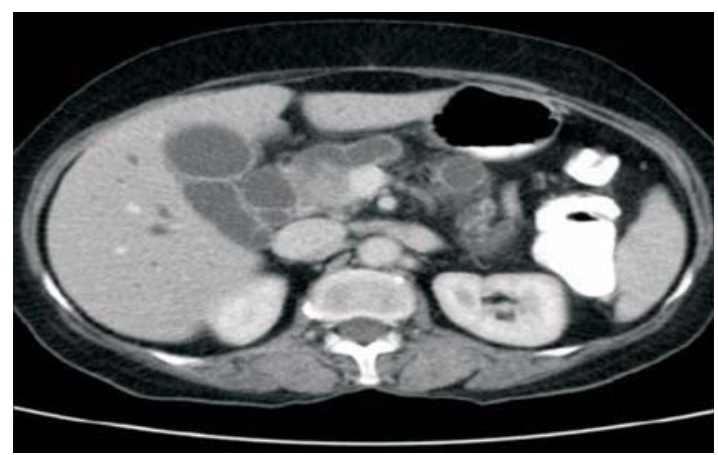

Fig. 1 Abdominal computed tomogram showing a pancreatic mass involving the head and uncinate process with dilated biliary and pancreatic ducts

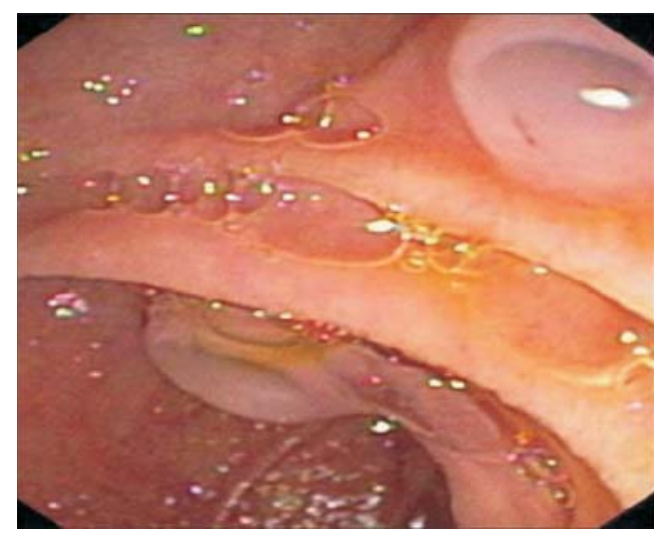

Fig. 2 Side-viewing duodenoscopy showing the classic "fish-eye appearance" of both the major and minor papillae

E. Simon $(\square)$ e-mail: ebbysimon@gmail.com

\section{References}

1. Ardengh JC, Lopes CV, Campos AD, Pereira de Lima LF, Venco F, Módena JL. Endoscopic ultrasound and fine needle aspiration in chronic pancreatitis: differential diagnosis between pseudotumoral masses and pancreatic cancer. JOP 2007; 8:413-21.

2. Okabayashi T, Kobayashi M, Nishimori I, et al. Clinicopathological features and medical management of intraductal papillary mucinous neoplasms. J Gastroenterol Hepatol 2006; 21:462-7. 